GEOMETRY IN NONLINEAR CONTROL

AND DIFFERENTIAL INCLUSIONS

BANACH CENTER PUBLICATIONS, VOLUME 32

INSTITUTE OF MATHEMATICS

POLISH ACADEMY OF SCIENCES

WARSZAWA 1995

\title{
VARIATIONAL APPROACH TO SOME OPTIMIZATION CONTROL PROBLEMS
}

\author{
R. M. BIANCHINI \\ Department of Mathematics U. Dini, University of Florence, \\ Viale Morgagni 67/A, 50134 Firenze, Italy \\ E-mail:rosabian@udini.math.unifi.it
}

\begin{abstract}
This paper presents the variational approach to some optimization problems: Mayer's problem with or without constraints on the final point, local controllability of a trajectory, time-optimal problems.
\end{abstract}

1. Introduction. Reachable sets of control systems are usually very difficult to compute. In dealing with optimization problems, a way to overcome this difficulty consists in approximating the reachable set in a neighborhood of the final point of the optimal trajectory by means of tangent vectors of the reachable set. In this paper we want to review the use of tangent vectors in studying some optimization problems. We will show that only subsets of tangent vectors with suitable properties can be used and we will single out what kind of properties are required to obtain necessary or sufficient conditions for the following problems: the Mayer optimization problem with or without constraints on the final point, the minimum time problem, the local controllability problem.

The motivation of this investigation is the search for a good definition of trajectory variation. Given a reference trajectory $x^{*}$ of a control system, a variation of $x^{*}$ is a tangent vector, $v$, at $x^{*}(t)$ such that the transport of $v$ along the reference flow from time $t$ up to the final time, $t_{1}$, is a tangent vector of the reachable set at $x^{*}\left(t_{1}\right)$. There are several definitions of variation of $x^{*}$ (see [2] and the references therein); in general they are very technical and it is difficult to compare them. Therefore it is natural to look for a definition of variation which is simple,

1991 Mathematics Subject Classification: 49B10, 49E15.

Key words and phrases: tangent vectors, variational cone, Mayer's optimization problem, local controllability.

Research supported by a grant from Ministero dell'Università e della Ricerca Scientifica e Tecnologica.

The paper is in final form and no version of it will be published elsewhere. 
but provides sufficient information on the reachable sets in order to be used in optimization theory. The starting point for this goal is to single out what kind of information one needs in studying a particular problem.

The paper is organized as follows: in Section 2 we recall the definition of the tangent cone of a subset of an n-dimensional manifold and the definitions of subsets of it which have been used in optimization theory. In spite of the different definitions, derived subsets and subsets of tangent vectors which can be continuously summed are the same object; more general subsets are regular tangent cones. These subsets of tangent vectors have been introduced by Polovinkin and Smirnov and share important properties with the other subsets. In Section 3 we review some standard optimization control problems and their variational approach. Our point of view unifies and generalizes the known theory. It points out the interest in determining regular tangent cones of the reachable set at the final point of a trajectory. Section 4 is devoted to the construction of a cone of this type. We do not provide proofs. These will appear in a forthcoming paper. The cone presented here is a generalization of the one in [2] and it provides high order Maximum Principles. This topic however is beyond the scope of this paper.

The following notations will be used throughout the paper. $M$ denotes a dififerentiable manifold.

Given $x \in M$, we will denote by $o(\epsilon)$ a map defined on an Euclidian space with values in $T_{x} M$ such that $\lim _{\epsilon \rightarrow 0} \frac{o(\epsilon)}{\|\epsilon\|}=0$.

For $v \in T_{x} M$, we will write

$$
y=x+\epsilon v+o(\epsilon)
$$

if the equality holds in a chart at $x$ and hence in any chart.

The symbols int $K, c l K, \partial K$ denote respectively the interior, the closure and the boundary of a subset $K$ of a topological space $X$. In the case where $X$ is a vector space, $c o K$ denotes the convex hull of $K$.

Given a convex subset $K$ of $T_{x} M$, we denote by $i n t_{r e l} K$ the relative interior of $K$. B stands for the unit closed ball of $T_{x} M$ and $D g(y)$ for the derivative of a map $g$ at the point $y$.

2. Tangent cones. Let $Y$ be a subset of a $C^{1} n$-dimensional manifold $M$ and let $x \in Y$.

Definition 2.1. A vector $v \in T_{x} M$ is a tangent vector of $Y$ at $x$ if

$$
x+\epsilon v+o(\epsilon) \in Y \text {. }
$$

The set of tangent vectors of $Y$ at $x$ is named the intermediate tangent cone of $Y$ at $x$ and it is denoted by $I_{x}(Y)$.

The intermediate tangent cone approximates $Y$ in a neighborhood of $x$ but for some problems it is too large to provide a "good" description of the set, for example $I_{x}(Y)$ may be equal to $T_{x} M$ even if $x$ is a boundary point of $Y$, see Example 3.1. Therefore we are interested in singling out particular subsets of 
$I_{x}(Y)$ which provide a more precise description of the set $Y$. Let us recall the definitions of some subsets used in optimization theory.

Definition 2.2. A cone $K \subset I_{x}(Y)$ is a derived cone [5] if for each finite subset $\left\{v_{1}, \ldots, v_{p}\right\} \subset K$ there exists a positive number $\delta^{\prime}$ and a continuous map $d:\left[0, \delta^{\prime}\right]^{p} \rightarrow T_{x} M$ such that $d(\delta)=o(\delta)$ and

$$
x+\sum_{i=1}^{p} \delta_{i} v_{i}+d(\delta) \in Y .
$$

Definition 2.3. A cone $K \subset I_{x}(Y)$ is a cone of tangent vectors which can be continuously summed [6] if for each finite subset $\left\{v_{1}, \ldots, v_{p}\right\} \subset K$ there exist positive numbers $\bar{c}, \bar{\epsilon}$ and a continuous map $s:[0, \bar{\epsilon}] \times[0, \bar{c}]^{p} \rightarrow T_{x} M$ such that $s(\epsilon, c)=o(\epsilon)$ uniformly with respect to $c$ and

$$
x+\epsilon \sum_{i=1}^{p} c_{i} v_{i}+s(\epsilon, c) \in Y .
$$

Definition 2.4. A cone $K \subset I_{x}(Y)$ is a regular tangent cone [7] if for each $\epsilon \in[0, \bar{\epsilon}]$ there exists a continuous map $r_{\epsilon}: \operatorname{co} K \cap B \rightarrow T_{x} M$ such that $\lim _{\epsilon \rightarrow 0^{+}}\left\|r_{\epsilon}(y)\right\| / \epsilon=0$ uniformly with respect to $y$ and

$$
x+\epsilon y+r_{\epsilon}(y) \in Y \quad \forall y \in \operatorname{co} K \cap B .
$$

A cone of tangent vectors $K$ is said a local regular tangent cone of $Y$ at $x$ if for each vector $v \in i n t_{r e l}$ co $K$ there exists a neighborhood $W$ of $v$ such that $W \cap K$ is a regular tangent cone of $Y$ at $x$.

Derived cones and cones of tangent vectors which can be continuously summed are not different objects. In fact we have:

Proposition 2.1. $K$ is a derived cone if and only if it is a set of tangent vectors which can be continuously summed.

Pr o of. Let us suppose that $K$ is a derived cone and let $\left\{v_{1}, \ldots, v_{p}\right\} \subset K$. By definition there exists a continuous map $d:[0, \bar{\delta}]^{p} \rightarrow T_{x} M$ such that $d(\delta)=o(\delta)$ and

$$
x+\sum_{i=1}^{p} \delta_{i} v_{i}+d(\delta) \in Y .
$$

Set $s:[0, \sqrt{\bar{\delta}}] \times[0, \sqrt{\bar{\delta}}]^{p} \rightarrow T_{x} M, s(\epsilon, c)=d\left(\epsilon c_{1}, \ldots, \epsilon c_{p}\right)$. By construction $s(\epsilon, c)=$ $o(\epsilon)$ uniformly with respect to $c$ and

$$
x+\epsilon \sum_{i=1}^{p} c_{i} v_{i}+s(\epsilon, c) \in Y
$$

so that $K$ is a set of tangent vectors which can be continuously summed.

Let $K$ be a set of tangent vectors which can be continuously summed and let $\left\{v_{1}, \ldots, v_{p}\right\} \subset K$. Let $\bar{\epsilon}, \bar{c}$ and $s$ be as in Definition 2.3. Let $\bar{\delta}=\overline{c \epsilon} / \sqrt{p}$; set 
$d:[0, \bar{\delta}]^{p} \rightarrow T_{x} M$,

$$
\begin{aligned}
d(\delta) & =s(\|\delta\| / \bar{c}, \bar{c} \delta /\|\delta\|), \quad \delta \neq 0, \\
d(0) & =0 .
\end{aligned}
$$

$d$ is a continuous map such that $d(\delta)=o(\delta)$ and

$$
x+\sum_{i=1}^{p} \delta_{i} v_{i}+d(\delta) \in Y .
$$

Therefore $K$ is a derived set of tangent vectors.

By definition a cone of tangent vectors which can be continuously summed is a local regular tangent cone, therefore the regular tangent cone is the most general cone among the three defined above. Regular tangent cones have the important property that the points interior to their convex hull pick out subsets of $Y$.

Definition 2.5. A vector $v \in I_{x}(Y)$ is a regular tangent direction [3] of $Y$ if there exists $\bar{\epsilon}>0$ such that

$$
x+\delta(v+\bar{\epsilon} B) \subset Y, \quad \forall \delta \in[0, \bar{\epsilon}] .
$$

Proposition 2.2. The points belonging to the interior of the convex hull of a regular tangent cone are regular tangent directions.

This property is probably known, but for the sake of completeness I am going to sketch its proof.

Pro of. Since all the properties we are dealing with are local, we can suppose that $M=R^{n}$. Let $K$ be a regular tangent cone and let $v \in$ int $\operatorname{co} K$. There exists $\left\{v_{1}, \ldots, v_{p}\right\} \subset K \cap B$ and $\alpha>0$ such that

$$
\alpha v=w \in \operatorname{int} \operatorname{co}\left\{v_{1}, \ldots, v_{p}\right\}=\text { int } H .
$$

Let $\bar{\delta}$ be such that $w+\bar{\delta} B \subset$ int $H$ and let $d>0$ be less than the distance between $w+\bar{\delta} B$ and $\partial H$. By definition of regular tangent cone, for all $\epsilon$ sufficiently small, say $\epsilon<a$, there exists a continuous map $r_{\epsilon}: H \rightarrow T_{x} M=R^{n}$ such that $\lim _{\epsilon \rightarrow 0^{+}}\left\|r_{\epsilon}(y)\right\| / \epsilon=0$ uniformly with respect to $y \in H$ and

$$
x+\epsilon y+r_{\epsilon}(y) \in Y, \quad \forall y \in K .
$$

Choose $\bar{d}$ such that $\left\|r_{\epsilon}(y)\right\| / \epsilon<d, \forall \epsilon \in[0, \bar{d}]$ and $\forall y \in H$. Let $f_{\epsilon}: H \rightarrow R^{n}$ be defined by

$$
\begin{aligned}
& f_{\epsilon}(y)=y+r_{\epsilon}(y) / \epsilon, \quad \epsilon \neq 0, \\
& f_{0}(y)=y .
\end{aligned}
$$

Then $f_{\epsilon}$ is a continuous map. Let $z \in w+\bar{\delta} B$ and let $u \in \partial H$. If $\epsilon<\min \{a, \bar{d}\}$ then

$$
\left\|f_{\epsilon}(u)-u\right\|=\left\|r_{\epsilon}(u)\right\| / \epsilon<d<\|z-u\| .
$$


By the scolium lemma, [6, page 251], $z \in f_{\epsilon}(H)$, that is there exists $b \in H$ such that $z=b+r_{\epsilon}(b) / \epsilon$. Since

$$
x+\epsilon z=x+\epsilon b+r_{\epsilon}(b) \in Y
$$

the property is proved.

There is another subcone of $I_{x}(Y)$ which has the property that its interior points are regular tangent directions: the Clarke tangent cone.

Definition 2.6. A vector $v \in T_{x} M$ is a Clarke tangent vector of $Y$ at $x$ if for all sequences $\left\{\epsilon_{n}\right\} \subset R^{+}$converging to 0 and all sequences $\left\{x_{n}\right\} \subset Y$ converging to $x$, there exists a sequence $\left\{v_{n}\right\} \subset T_{x} M$ converging to $v$ such that

$$
x_{n}+\epsilon_{n} v_{n} \in Y \text {. }
$$

The set of Clarke tangent vectors of $Y$ at $x$ is the Clarke tangent cone.

Proposition 2.3 [8]. The Clarke tangent cone of $Y$ at $x$ is a convex cone whose interior points are regular tangent directions of the closure of $Y$ at $x$.

The Clarke tangent cone is very different from the other ones defined above; it is a convex cone which provides information on the boundary of the closure of $Y$ near $x$. However the Clarke tangent cones of reachable sets of a control system are difficult to compute.

Definition 2.5, Propositions 2.2 and 2.3 imply the following important property:

Proposition 2.4. A point $x$ is an interior point of $Y$ if and only if there exists a local regular tangent cone $K \subset I_{x}(Y)$ whose convex hull coincides with $T_{x} M$. If there exists a set of Clarke tangent vectors of $Y$ at $x$ whose convex hull coincides with $T_{x} M$, then $x$ is interior to the closure of $Y$.

In dealing with some optimization control problems it is important to obtain information on the intersection of two sets from the tangent vectors of the two sets at a common point. Regular tangent cones are suitable for this purpose.

Proposition 2.5. Let $Y_{1}, Y_{2} \subset M$ and $x \in Y_{1} \cap Y_{2}$; if $N_{1}, N_{2}$ are local regular tangent cones respectively of $Y_{1}$ and $Y_{2}$ at $x$ which cannot be separated, then

$$
\emptyset \neq i n t_{r e l} \operatorname{co} N_{1} \cap i n t_{r e l} \text { co } N_{2} \subset I_{x}\left(Y_{1} \cap Y_{2}\right) .
$$

Proof. By the properties of convex sets, $N_{1}$ not separable from $N_{2}$ implies int $_{r e l}$ co $N_{1} \cap i n t_{r e l}$ co $N_{2} \neq \emptyset$ and span $N_{1} \cup N_{2}=T_{x} M$. Without loss of generality we can suppose that $N_{1}, N_{2}$ are regular tangent cones. Let $v \in i n t_{\text {rel }} \operatorname{co} N_{1} \cap$ int $_{\text {rel }} \operatorname{co} N_{2},\|v\|<1$; there exist $\left\{v_{1}, \ldots, v_{p}\right\} \subset N_{1} \cap B,\left\{w_{1}, \ldots, w_{s}\right\} \subset N_{2} \cap B$ and non-negative numbers $a_{1}, \ldots, a_{p}, b_{1}, \ldots, b_{s}$ such that $\left(v_{1}, \ldots, v_{p}, w_{1}, \ldots, w_{s}\right)$ contains a basis of $T_{x} M$ and $v=\sum_{i=1}^{p} a_{i} v_{i}=\sum_{j=1}^{s} b_{j} w_{j}$. We can suppose that $\left\{v_{1}, \ldots, v_{m}, w_{1}, \ldots, w_{l}\right\}$ is a basis for $T_{x} M$. By definition of regular tangent cone there exist $\bar{\epsilon}$ and for all $\epsilon \in[0, \bar{\epsilon}]$ two continuous maps $g_{\epsilon}: \operatorname{co} N_{1} \cap B \rightarrow T_{x} M$, 
$h_{\epsilon}: \operatorname{co} N_{2} \cap B \rightarrow T_{x} M$ such that

$$
x+\epsilon y+g_{\epsilon}(y) \in Y_{1} \quad \text { and } \quad x+\epsilon y+h_{\epsilon}(y) \in Y_{2} .
$$

Let $\bar{\delta}$ be such that $\forall d_{i}, c_{j} \in[-\bar{\delta}, \bar{\delta}]$,

$$
\begin{gathered}
\sum_{i=1}^{m}\left(a_{i}+d_{i}\right) v_{i}+\sum_{i=m+1}^{p} a_{i} v_{i} \in \operatorname{co} N_{1} \cap B \text { and } \\
\sum_{j=1}^{l}\left(b_{j}+c_{j}\right) w_{j}+\sum_{j=l+1}^{s} b_{j} w_{j} \in \operatorname{co} N_{2} \cap B .
\end{gathered}
$$

For all $\delta \in(0, \bar{\delta}]$, let

$$
W_{\delta}=\left\{\sum_{i=1}^{m} d_{i} v_{i}+\sum_{j=1}^{l} c_{j}\left(-w_{j}\right)\right\}, \quad d_{i}, c_{j} \in[-\delta, \delta] .
$$

$W_{\delta}$ is a compact convex subset of $T_{x} M$ which has 0 as an interior point. Let $d(\delta)=\min \|w\|, w \in \partial W_{\delta} ; d(\delta)$ is a positive number. By the properties of $g_{\epsilon}$ and $h_{\epsilon}$ it follows that there exists $\sigma(\delta)$ such that, for all $\epsilon \in[0, \sigma(\delta)], \forall u \in \operatorname{co} N_{1} \cap B$, $\forall v \in \operatorname{co} N_{2} \cap B,\left\|g_{\epsilon}(u)\right\| / \epsilon$ and $\left\|h_{\epsilon}(v)\right\| / \epsilon$ are less than $d(\delta) / 2$. Set $\delta(\epsilon)=\inf \{\delta$ : $\epsilon \leq \sigma(\delta)\}$. $\delta(\cdot)$ is a non-decreasing map; let $a=\lim _{\epsilon \rightarrow 0^{+}} \delta(\epsilon)$. If $a>0$, then $0<\sigma(a / 2)<\epsilon, \forall \epsilon>0$, an absurd. Therefore $\delta(\epsilon)$ goes to zero with $\epsilon$. For all $\epsilon \in[0, \bar{\epsilon}]$, let $f_{\epsilon}: W_{\delta(\epsilon)} \rightarrow T_{x} M$ be the map defined by:

$$
\begin{aligned}
f_{0}\left(\sum_{i=1}^{m} d_{i} v_{i}-\sum_{j=1}^{l} c_{j} w_{j}\right)= & \sum_{i=1}^{m} d_{i} v_{i}-\sum_{j=1}^{l} c_{j} w_{j} \\
f_{\epsilon}\left(\sum_{i=1}^{m} d_{i} v_{i}-\sum_{j=1}^{l} c_{j} w_{j}\right)= & \sum_{i=1}^{m} d_{i} v_{i}-\sum_{j=1}^{l} c_{j} w_{j} \\
& +(1 / \epsilon) g_{\epsilon}\left(\sum_{i=1}^{m}\left(a_{i}+d_{i}\right) v_{i}+\sum_{i=m+1}^{p} a_{i} v_{i}\right) \\
& -(1 / \epsilon) h_{\epsilon}\left(\sum_{j=1}^{l}\left(b_{j}+c_{j}\right) w_{j}+\sum_{j=l+1}^{s} b_{j} w_{j}\right) \\
=f_{\varepsilon}(w)= & w+(1 / \epsilon)\left(g_{\epsilon}(v(w))-h_{\epsilon}(u(w))\right) .
\end{aligned}
$$

By construction $f_{\epsilon}$ is a continuous map. For all $w \in \partial W_{\delta(\epsilon)}$

$$
\left\|f_{\epsilon}(w)-w\right\|=\frac{\left\|g_{\epsilon}(v(w))-h_{\epsilon}(u(w))\right\|}{\epsilon}<d(\delta(\epsilon)) \leq\|w\| .
$$

By the scolium lemma there exist $d_{i}(\epsilon), c_{j}(\epsilon),\left|d_{i}(\epsilon)\right| \leq \delta(\epsilon),\left|c_{j}(\epsilon)\right| \leq \delta(\epsilon)$, such 
that

$$
\begin{aligned}
& \frac{1}{\epsilon} g_{\epsilon}\left(\sum_{i=1}^{m}\left(a_{i}+d_{i}(\epsilon)\right) v_{i}+\sum_{i=m+1}^{p} a_{i} v_{i}\right) \\
& \left.\quad-\frac{1}{\epsilon} h_{\epsilon}\left(\sum_{j=1}^{l}\left(b_{j}+c_{j}(\epsilon)\right) w_{j}+\sum_{j=l+1}^{s} b_{j} w_{j}\right)\right)+\sum_{i=1}^{m} d_{i}(\epsilon) v_{i}-\sum_{j=1}^{l} c_{j}(\epsilon) w_{j}=0 .
\end{aligned}
$$

Therefore

$$
\begin{aligned}
Y_{1} \ni & x+\epsilon\left(v+\sum_{i=1}^{m} d_{i}(\epsilon) v_{i}\right)+g_{\epsilon}\left(\sum_{i=1}^{m}\left(a_{i}+d_{i}(\epsilon)\right) v_{i}+\sum_{i=m+1}^{p} a_{i} v_{i}\right) \\
& =x+\epsilon\left(v+\sum_{j=1}^{l} c_{j}(\epsilon) w_{j}\right)+h_{\epsilon}\left(\sum_{j=1}^{l}\left(b_{j}+c_{j}(\epsilon)\right) w_{j}+\sum_{j=l+1}^{s} b_{j} w_{j}\right) \in Y_{2} .
\end{aligned}
$$

Since $\delta(\epsilon)$ goes to zero with $\epsilon$, the proposition is proved.

Remark. For derived sets Proposition 2.5 has been proved by S. Mirica.

3. Control problems and tangent cones. Let us consider a control process on $M$

$$
\dot{x}=f(t, x, u)
$$

satisfying the following assumptions:

A. the control maps $u(\cdot)$ belong to an assigned subset $\mathcal{U}$ of the locally integrable maps from $R$ to a set $U \subset R^{m}$;

B. $J$ is an open interval of $R$ and $f: J \times M \times U \rightarrow T M$ is such that for all $u(\cdot) \in \mathcal{U}$ the time-dependent vector field $f(t, x, u(t))$ is a quasi- $C^{1}$ vector field, [4], i.e.

for almost all $t \in J, x \mapsto f(t, x, u(t))$ is $C^{1}$;

for each $x \in M, t \mapsto f(t, x, u(t))$ is measurable;

in each chart $(\varphi, U)$ of $M$ for each compact set $K \subset \varphi(U)$ there exists an integrable map $\psi$ such that

$$
\|f(t, x, u(t))\|+\left\|D_{x} f(t, x, u(t))\right\| \leq \psi(t) \quad \forall x \in K .
$$

Under these assumptions, for each $\left(t_{0}, x_{0}\right) \in J \times M$ and each $u \in \mathcal{U}$ there exists only one maximal solution of (1), $S\left(\cdot, t_{0}, x_{0}, u\right)$, such that $x_{0}=S\left(t_{0}, t_{0}, x_{0}, u\right)$.

Let $S_{0}$ be a subset of $M$; for all $\left(t_{0}, t\right) \in J \times J$ we will denote by $R\left(t, t_{0}, S_{0}\right)$ the set of points reachable at time $t$ by means of the solutions of (1) which at time $t_{0}$ belong to $S_{0}$, that is

$$
R\left(t, t_{0}, S_{0}\right)=\left\{x(t): x(\cdot) \text { solution of }(1) \text { such that } x\left(t_{0}\right) \in S_{0}\right\} .
$$

Notice that $R\left(t, t_{0}, S_{0}\right)$ may be the empty set for some $t$.

In this section we want to review some classical optimization control problems. Let us start with the Mayer control problem 
MP. Let $g_{0}: M \rightarrow R$ be a $C^{1}$ map and let $t_{1}>t_{0}$ be an assigned point of $J$. Find among the trajectories $x(\cdot)$ of (1) satisfying the condition $x\left(t_{0}\right) \in S_{0}$ a trajectory $x^{*}$ which minimize $g_{0}$ over $R\left(t_{1}, t_{0}, S_{0}\right)$, i.e.

$$
g_{0}\left(x^{*}\left(t_{1}\right)\right)=\min \left\{g_{0}(y): y \in R\left(t_{1}, t_{0}, S_{0}\right)\right\} .
$$

The intermediate tangent cone of $R\left(t_{1}, t_{0}, S_{0}\right)$ provides necessary conditions for a trajectory to solve the Mayer control problem. In fact it is easy to prove that

Proposition 3.1. If $x^{*}$ solves the Mayer problem, then

$$
D g_{0}\left(x^{*}\left(t_{1}\right)\right) v \geq 0, \quad \forall v \in I_{x^{*}\left(t_{1}\right)}\left(R\left(t_{1}, t_{0}, S_{0}\right)\right) .
$$

Different is the case in which there are constraints on the final point of the trajectory. The Mayer control problem with constraints on the end points is the following:

CMP. Let $S_{1}$ be a subset of $M$, find among the trajectories $x(\cdot)$ of (1) satisfying the conditions $x\left(t_{0}\right) \in S_{0}, x\left(t_{1}\right) \in S_{1}$ a trajectory $x^{*}$ which minimizes $g_{0}$ i.e.

$$
g_{0}\left(x^{*}\left(t_{1}\right)\right)=\min \left\{g_{0}(y), y \in S_{1} \cap R\left(t_{1}, t_{0}, S_{0}\right)\right\} .
$$

Notice that $I_{x^{*}\left(t_{1}\right)} R\left(t_{1}, t_{0}, S_{0}\right)$ may coincide with the whole tangent space even if $x^{*}$ solves the CMP problem. For example consider the following bilinear system on $R^{2}$ :

EXAMPLE 3.1.

$$
\begin{aligned}
& \left\{\begin{array}{l}
\dot{x}=u_{1}(-x-y+0.0278)+u_{2}(-x+y+0.0278)+u_{3}\left(139-10^{5} x\right), \\
\dot{y}=u_{1}(x-y)+u_{2}(-x-y),
\end{array}\right. \\
& \left(u_{1}, u_{2}, u_{3},\right) \in\{(0,0,0),(1,0,0),(0,1,0),(0,0,1)\} .
\end{aligned}
$$

The reachable set from $(0,(1.027,0))$ at time 3.92 is contained in a heartshaped set, $K$, whose boundary is the union of two arcs of spiral symmetric with respect to the $x$-axis. The point $(0,0)$ belongs to $R(3.92,0,(1.027,0))$ and it is the cupsidal point of the set $K$. The intermediate tangent cone of $R(3.92,0,(1.027,0))$ at $(0,0)$ is equal to the whole $R^{2}$ but if $S_{1}=\{(\alpha, 0): \alpha \in R\}$ and $g_{0}(x, y)=x$, then $(0,0)$ is a minimum.

A necessary condition for the CMP control problem can be deduced from the tangent vectors of the intersection of the reachable set with the set $S_{1}$; therefore Proposition 2.5 suggests the use of local regular tangent cones.

THEOREM 3.1. Let $x^{*}$ solves the CMP. If $\mathcal{K}$ is a local regular tangent cone of $R\left(t_{1}, t_{0}, S_{0}\right)$ at $x^{*}\left(t_{1}\right)$ and $\mathcal{N}$ is a local regular tangent cone of $S_{1}$ at $x^{*}\left(t_{1}\right)$, then there exist $\lambda_{0} \leq 0$ and a cotangent vector $\nu \in T_{x^{*}\left(t_{1}\right)}^{*} M$ such that

$$
\begin{gathered}
\nu w \geq 0, \quad \forall w \in \mathcal{N}, \\
\left(\lambda_{0} D g_{0}\left(x^{*}\left(t_{1}\right)\right)+\nu\right) v \leq 0, \quad \forall v \in \mathcal{K} .
\end{gathered}
$$


Proof. If $\mathcal{N}$ and $\mathcal{K}$ can be separated, then there exists $\nu \in T_{x^{*}\left(t_{1}\right)}^{*} M$ such that $\nu w \geq 0, \forall w \in \mathcal{N}$ and $\nu v \leq 0, \forall v \in \mathcal{K}$. The theorem is proved with $\lambda_{0}=0$. In this case the problem is called abnormal.

If $\mathcal{N}$ and $\mathcal{K}$ cannot be separated, then by proposition 2.5

$$
\emptyset \neq i n t_{r e l} \operatorname{co} \mathcal{N} \cap i n t_{r e l} \operatorname{co} \mathcal{K} \subset I_{x^{*}\left(t_{1}\right)}\left(S_{1} \cap R\left(t_{1}, t_{0}, S_{0}\right)\right) .
$$

Since $x^{*}$ solves the CMP, then

$$
D g_{0}\left(x^{*}\left(t_{1}\right)\right) w \geq 0, \quad \forall w \in I_{x^{*}\left(t_{1}\right)}\left(S_{1} \cap R\left(t_{1}, t_{0}, S_{0}\right)\right) .
$$

Therefore (2) implies that $-D g_{0}\left(x^{*}\left(t_{1}\right)\right)$ belongs to $(\mathcal{N} \cap \mathcal{K})^{*}$, the polar cone of $\mathcal{N} \cap \mathcal{K}$, that is

$$
-D g_{0}\left(x^{*}\left(t_{1}\right)\right) y \leq 0, \quad y \in \mathcal{N} \cap \mathcal{K} .
$$

But, see [1], $(\mathcal{N} \cap \mathcal{K})^{*}=(\mathcal{N})^{*}+(\mathcal{K})^{*}$, so that there exists $-\nu \in(\mathcal{N})^{*} \subset T_{x^{*}\left(t_{1}\right)}^{*} M$ such that $-D g_{0}\left(x^{*}\left(t_{1}\right)\right)+\nu \in(\mathcal{K})^{*}$, that is,

$$
\begin{gathered}
\nu w \geq 0, \quad w \in \mathcal{N}, \\
\left(-D g_{0}\left(x^{*}\left(t_{1}\right)\right)+\nu\right) v \leq 0, \quad \forall v \in \mathcal{K} .
\end{gathered}
$$

The proof is complete.

Corollary 3.1. Let $g_{i}, i=0, \ldots, p$, be $C^{1}$ maps from $M$ to $R$ such that $D g_{1}, \ldots, D g_{p}$ are linearly independent at any point of $M$. If $x^{*}\left(T_{1}\right)$ minimizes $g_{0}$ over the set $\left\{y \in R\left(t_{1}, t_{0}, S_{0}\right): g_{i}(y)=0, i=1, \ldots, p\right\}$ and $\mathcal{K}$ is a local regular tangent cone of $R\left(t_{1}, t_{0}, S_{0}\right)$ at $x^{*}\left(t_{1}\right)$ then there exists $\lambda=\left(\lambda_{0}, \ldots, \lambda_{p}\right) \neq$ $(0, \ldots, 0), \lambda_{0} \leq 0$ such that

$$
\sum_{i=0}^{p} \lambda_{i} D g_{i}\left(x^{*}\left(t_{1}\right)\right) v \leq 0, \quad \forall v \in \mathcal{K} .
$$

Proof. Let $S_{1}=\left\{y: g_{i}(y)=0, i=1, \ldots, p\right\}, S_{1}$ is a differentiable manifold. Our assumptions imply [5], that $T_{x^{*}\left(t_{1}\right)}\left(S_{1}\right)$ is a derived set of tangent vectors, and hence a regular tangent cone, of $S_{1}$ at $x^{*}\left(t_{1}\right)$. Since the polar cone of $T_{x^{*}\left(t_{1}\right)}\left(S_{1}\right)$ is the set

$$
\operatorname{span}\left\{D g_{i}\left(x^{*}\left(t_{1}\right)\right): i=1, \ldots, p\right\}
$$

Theorem 3.1 proves the statement.

Let us consider now the local controllability problem.

LC. A trajectory $x^{*}$ is locally controllable in the interval $\left[t_{0}, t_{1}\right] \subset J$ if

$$
x^{*}\left(t_{1}\right) \in \operatorname{int} R\left(t_{1}, t_{0}, x^{*}\left(t_{0}\right)\right) .
$$

Example 3.1 shows that a trajectory $x^{*}$ may be not locally controllable in $\left[t_{0}, t_{1}\right]$ even if $I_{x^{*}\left(t_{1}\right)}\left(R\left(t_{1}, t_{0}, x^{*}\left(t_{0}\right)\right)\right)=T_{x^{*}\left(t_{1}\right)} M$. To obtain sufficient conditions of local controllability of a trajectory one has to look for sets of tangent vectors which pick out regular tangent directions. Notice that for this property the separation property of Proposition 2.5 is not required. 
Proposition 3.2. A trajectory $x^{*}$ is locally controllable in the interval $\left[t_{0}, t_{1}\right]$ if either there exists a local regular tangent cone of $R\left(t_{1}, t_{0}, x^{*}\left(t_{0}\right)\right)$ at $x^{*}\left(t_{1}\right)$ whose convex hull contains zero as an interior point, or int $R\left(t_{1}, t_{0}, x^{*}\left(t_{0}\right)\right)=$ int $\mathrm{cl} R\left(t_{1}, t_{0}, x^{*}\left(t_{0}\right)\right)$ and there exists a set of Clarke tangent vectors whose convex hull contains zero as an interior point.

Let us consider the time-optimal problem.

OT. A trajectory $x^{*}$ is time-optimal in the interval $\left[t_{0}, t_{1}\right]$ if

$$
x^{*}\left(t_{1}\right) \notin R\left(t, t_{0}, S_{0}\right), \quad \forall t, t_{0} \leq t<t_{1} .
$$

If the control process $((1))$ is autonomous, i.e. $f$ does not depend on $t$ and if $u(\cdot) \in \mathcal{U}$ implies $u_{\tau}=u(\cdot+\tau) \in \mathcal{U}$ for any $\tau$, then $x^{*}$ is time-optimal only if $x^{*}(t) \in \partial R\left(t, t_{0}, S_{0}\right), \forall t \in\left[t_{0}, t_{1}\right)$. In fact if there exists $t$ such that $x^{*}(t) \in$ $\operatorname{int} R\left(t, t_{0}, S_{0}\right)$, then

$$
x^{*}(t+\epsilon) \in R\left(t, t_{0}, S_{0}\right)
$$

for $\epsilon$ sufficiently small. But (3) implies $x^{*}\left(t_{1}\right)=S\left(t_{1}-\epsilon, t, x^{*}(t+\epsilon), u_{\epsilon}^{*}\right) \in$ $R\left(t_{1}-\epsilon, t_{0}, S_{0}\right)$, a contradiction. Therefore by Proposition 3.2 we get:

Proposition 3.3. Let the process (1) be autonomous and let $\mathcal{U}$ be such that for each $u \in \mathcal{U}$ and each $\tau$ the map $u_{\tau}: t \mapsto u(t+\tau)$ belongs to $\mathcal{U}$. If $x^{*}$ is time-optimal in the interval $\left[t_{0}, t_{1}\right], t \in\left(t_{0}, t_{1}\right)$ and $K$ is a local regular tangent cone of $R\left(t, t_{0}, S_{0}\right)$ at $x^{*}(t)$, then there exists a cotangent vector $\lambda \in T_{x^{*}(t)}^{*} M$ such that:

$$
\lambda v \leq 0, \quad \forall v \in K
$$

4. Variational cone of a reference trajectory. Let $x^{*}$ be a given trajectory of (1) such that $x^{*}\left(t_{0}\right) \in S_{0}$. In the previous section we have shown that local regular tangent cones of $R\left(t, t_{0}, S_{0}\right)$ are of great interest in solving some optimization control problems. In this section we want to describe a way to construct a local regular tangent cone of $R\left(t, t_{0}, S_{0}\right)$ at the point $x^{*}(t)$. The cone we are going to present is a generalization of the one introduced by G. Stefani and myself in [2]. We will give only the main results without proofs. The proofs will be given in a forthcoming paper.

Let $x^{*}:\left[t_{0}, t_{1}\right] \rightarrow M$ be a trajectory of the system (1) relative to the control $u^{*}$ and such that $x^{*}\left(t_{0}\right) \in S_{0}$. The first step for defining a local regular tangent cone of $R\left(t, t_{0}, S_{0}\right)$ at $x^{*}(t)$ consists in defining variations of $x^{*}$ at any time $\tau \in\left[t_{0}, t\right]$. We recall from section 3 that $S\left(t, t_{0}, x_{0}, u\right)$ stands for the value at time $t$ of the solution of (1) relative to the control $u$ which at time $t_{0}$ is equal to $x_{0}$.

Definition 4.1. A vector $v \in T_{x^{*}(\tau)} M$ is a right variation of $x^{*}$ at time $\tau \in\left[t_{0}, t\right)$ if there are positive numbers $k, \bar{c}, \bar{\epsilon}$ and a two-parameter control variation

$$
\eta:[0, \bar{c}] \times[0, \bar{\epsilon}] \rightarrow \mathcal{U}
$$


such that the map

$$
(\xi, c, \epsilon) \mapsto S\left(\tau+2 \epsilon^{\frac{1}{k}}, \tau+\epsilon^{\frac{1}{k}}, \xi, \eta(c, \epsilon)\right)
$$

is continuous and continuously differentiable with respect to $\xi$ in a neighborhood of $x^{*}$ and

$$
S\left(\tau+2 \epsilon^{\frac{1}{k}}, \tau+\epsilon^{\frac{1}{k}}, x^{*}\left(\tau+\epsilon^{\frac{1}{k}}\right), \eta(c, \epsilon)=x^{*}\left(\tau+2 \epsilon^{\frac{1}{k}}\right)+\epsilon c v+o(\epsilon)\right.
$$

uniformly with respect to $c$.

Left variations at time $\tau \in\left(t_{0}, t_{1}\right]$ can be defined in an analogous way. The Pontryagin variations $[6]$ are examples of such a type of variation.

Proposition 4.1. Let $\mathcal{U}$ contain the constant maps with values in $U$. If $\tau$ is a Lebesgue point of the map $t \mapsto f\left(t, x^{*}(t), u^{*}(t)\right)$ and $\omega \in U$ is such that the map $t \mapsto S\left(t, \tau, x^{*}(\tau), \omega\right)$ is right derivable, then

$$
f\left(\tau, x^{*}(\tau), \omega\right)-f\left(\tau, x^{*}(\tau), u^{*}(\tau)\right)
$$

is both a right and a left variation of $\left(x^{*}, u^{*}\right)$ at $\tau$.

In [2] it is proved that the variations at $\tau$ are local objects at $x^{*}(\tau)$; in the same paper variations of the trajectory relative to the drift term of an affine $C^{\infty}$ control system are constructed using the relations in the Lie algebra associated to the system at the point $x^{*}(\tau)$.

The second step consists in transporting the variations at $\tau$ from the time $\tau$ to the time $t$ by means of the reference flow in order to obtain a tangent vector of $R\left(t, t_{0}, S_{0}\right)$ at $x^{*}(t)$. By assumption $\mathrm{B}$ the reference flow, that is, the map $(t, \tau, \xi) \mapsto S\left(t, \tau, \xi, u^{*}\right)$, is differentiable with respect to $\xi$ at any point $\left(t, \tau, x^{*}(\tau)\right)$. Let

$$
G(t, \tau)=\frac{\partial S}{\partial \xi}\left(t, \tau, x^{*}(\tau), u^{*}\right) .
$$

It is known, [4], that $G(t, \tau)$ is the solution at time $t$ of the linear differential equation

$$
\frac{d}{d t} G(t)=\frac{\partial f}{\partial x}\left(t, x^{*}(t), u^{*}(t)\right) G(t), \quad G(\tau)=I d .
$$

Definition 4.2. Let $t \in\left[t_{0}, t_{1}\right]$. The variational cone, $\mathcal{K}(t)$, of $\left(x^{*}, u^{*}\right)$ at time $t$ is the set

$$
\mathcal{K}(t)=\left\{G(t, \tau) v_{\tau}: v_{\tau} \text { variation at } \tau, \tau \in\left[t_{0}, t\right]\right\} .
$$

Notice that we take only right variations at $t_{0}$ and only left variations at $t_{1}$.

The main property of the variational cone is the following:

THEOREM 4.1. $\mathcal{K}(t)$ is a set of tangent vectors of $R\left(t, t_{0}, S_{0}\right)$ at $x^{*}(t)$ which can be continuously summed and therefore it is a local regular tangent cone. 
Definition 4.2 and Proposition 4.1 imply that $\mathcal{K}(t)$ contains the Pontryagin tangent vectors

$$
G(t, \tau)\left(f\left(\tau, x^{*}(\tau), \omega\right)-f\left(\tau, x^{*}(\tau), u^{*}(\tau)\right)\right) \in \mathcal{K}(t) .
$$

Therefore Proposition 4.1, Theorem 4.1 and the results of the previous section imply that the cone $\mathcal{K}(t)$ can be used for stating high order maximum principles for Mayer control problems and for the time-optimal problem.

The variational cone $\mathcal{K}(t)$ can be used also for obtaining sufficient condition of local controllability of a reference trajectory; in [2] it has been used for proving a sufficient condition which unifies and generalizes almost all the ones previously known.

\section{References}

[1] M. S. Bazaraa and C. M. Shetty, Foundations of Optimization, Lecture Notes in Econom. and Math. Systems 122, Springer, Berlin, 1976.

[2] R. M. Bianchini and G. Stefani, Controllability along a reference trajectory: a variational approach, SIAM J. Control Optim. 31 (1993), 900-927.

[3] A. I. Dubovickiŭ and A. M. Miljutin, Extremum problems with constraints, J. Soviet Math. 4 (1963), 452-455.

[4] K. Grasse, Controllability and accessibility in nonlinear control systems, PhD thesis, University of Illinois at Urbana-Champaign, 1979.

[5] M. R. Hestenes, Calculus of Variations and Optimal Control Theory, Wiley, New York, 1966.

[6] E. B. Lee and L. Markus, Foundations of Optimal Control Theory, Wiley, New York, 1967.

[7] E. S. Polovinkin and G. V. Smirnov, An approach to the differentiation of manyvalued mappings and necessary conditions for optimization of solutions of differential inclusions, Differencial'nye Uravnenija 22 (1986), 944-954.

[8] R. T. Rockafellar, Clarke's tangent cones and the boundaries of closed convex sets in $R^{n}$, Nonlinear Anal. 3 (1979), 145-154. 